General Letters in Mathematics Vol. 6, No. 2, June 2019, pp.45-60

e-ISSN 2519-9277, p-ISSN 2519-9269

Available online at http:// www.refaad.com

https://doi.org/10.31559/glm2019.6.2.1

\title{
Sequence Spaces Defined by Fibonacci Matrix
}

\author{
M. KÜÇÜKASLAN ${ }^{* 1}$, B. ARIS ${ }^{2}$ \\ ${ }^{1}$ Mersin University Faculty of Science, Department of Mathematics 33343 Mersin, TURKEY. \\ 2 Istanbul University Faculty of Science, Department of Mathematics 34452 Istanbul, TURKEY. \\ ${ }^{1}$ mkucukaslan@mersin.edu.tr, ${ }^{2}$ busra.unal@istanbul.edu.tr
}

Abstract. In this paper, by using well known Fibonacci numbers, so far not described in the literature a new regular matrix $F=\left(f_{n k}\right)$ is defined and compared with well known matrix transformations. By using this new matrix, Fibonacci sequence space $c_{0}(F), c(F), l_{\infty}(F)$ and $l_{p}(F)(1 \leq p<\infty)$ are introduced. In addition to examining the properties of the new sequence spaces some results which contains comparison of $l_{p}(F)(1 \leq p<\infty)$ with other summability methods are given. Finally, $\alpha, \beta$ and $\gamma$ duals of $c_{0}(F), c(F), l_{\infty}(F)$ are characterized.

Keywords: Fibonacci numbers, Matrix transformation, Regular matrix, Sequence space, Summability method. 2010 MSC No: 40A05,40C05, 40D05

\section{Introduction}

Let $w$ be the space of all real valued sequences. Each subspaces of $w$ is called a sequence space. The subspaces $c_{0}, c, l_{p}(1 \leq p<\infty)$ and $l_{\infty}$ are the sequence spaces of null, convergent, p-absolutely convergent series and bounded, respectively. That is,

$$
\begin{aligned}
& c_{0}:=\left\{x=\left(x_{k}\right) \in w: \lim _{k \rightarrow \infty} x_{k}=0\right\} \\
& c:=\left\{x=\left(x_{k}\right) \in w: \lim _{k \rightarrow \infty} x_{k} \text { exists }\right\}
\end{aligned}
$$

and

$$
\begin{aligned}
& l_{p}:=\left\{x=\left(x_{k}\right) \in w: \sum_{k=1}^{\infty}\left|x_{k}\right|^{p}<\infty\right\}, \quad(1 \leq p<\infty), \\
& l_{\infty}:=\left\{x=\left(x_{k}\right) \in w: \sup _{k \in \mathbb{N}}\left|x_{k}\right|<\infty\right\} .
\end{aligned}
$$

\footnotetext{
${ }^{*}$ Corresponding author. M. KÜÇÜKASLAN and B. ARIS ${ }^{1}$ mkucukaslan@mersin.edu.tr
} 
The $\alpha, \beta$ and $\gamma$ duals of a sequence space $X \subset w$ are respectively defined as follow:

$$
\begin{aligned}
& X^{\alpha}:=\left\{a=\left(a_{k}\right) \in w:\left|\sum_{k} a_{k} x_{k}\right| \text { is convergent, } \forall x=\left(x_{k}\right) \in X\right\}, \\
& X^{\beta}:=\left\{a=\left(a_{k}\right) \in w: \sum_{k} a_{k} x_{k} \text { is convergent, } \forall x=\left(x_{k}\right) \in X\right\}, \\
& X^{\gamma}:=\left\{a=\left(a_{k}\right) \in w: \sum_{k} a_{k} x_{k} \text { is bounded, } \forall x=\left(x_{k}\right) \in X\right\} .
\end{aligned}
$$

Let $X$ and $Y$ be two sequence spaces and let $A=\left(a_{n k}\right)$ be an infinite matrix such that $a_{n k} \in \mathbb{R}$, for all $n, k \in \mathbb{N}$. expression

We recall the matrix mapping by $A: X \mapsto Y$, for every $x=\left(x_{k}\right)$ in $X, A x=\left(A_{n}(x)\right)$ in $Y$. The following

$$
A_{n}(x):=\sum_{k=1}^{\infty} a_{n k} x_{k}, \quad n \in \mathbb{N},
$$

is called the $A$-transformation of the sequence $x=\left(x_{k}\right)$. Moreover, the domain $X_{A}$ of a matrix $A$ is defined by

$$
X_{A}=\{x \in w: A x \in X\} .
$$

It is known that if $A$ is an infinite triangle matrix, then the spaces $X_{A}$ and $X$ are isometrically isomorphic [2].

Now, let $A=\left(a_{n k}\right)$ be an infinite matrix and following conditions are given

$$
\begin{gathered}
\sup _{n \in \mathbb{N}} \sum_{k}\left|a_{n k}\right|<\infty, \\
\lim _{n \rightarrow \infty} a_{n k}=0 \text { for each } k \in \mathbb{N}, \\
\exists \alpha_{k} \in \mathbb{C}, \quad \lim _{n \rightarrow \infty} a_{n k}=\alpha_{k} \text { for each } k \in \mathbb{N}, \\
\lim _{n \rightarrow \infty} \sum_{k} a_{n k}=0, \\
\exists \alpha \in \mathbb{C}, \lim _{n \rightarrow \infty} \sum_{k} a_{n k}=\alpha, \\
\sup _{K \subset F} \sum_{n}\left|\sum_{k \in K} a_{n k}\right|<\infty,
\end{gathered}
$$

where $F$ finite subset of $\mathbb{N}[\underline{5}$.

The following Lemma characterization of the matrix transformation was given between some sequence spaces.

Lemma 1.1. [15] The following statements hold:

(a) $A=\left(a_{n k}\right) \in\left(c_{0}, c_{0}\right)$ if and only if (1) and (2) hold.

(b) $A=\left(a_{n k}\right) \in\left(c_{0}, c\right)$ if and only if (1) and (3) hold.

(c) $A=\left(a_{n k}\right) \in\left(c, c_{0}\right)$ if and only if (1), (2) and (4) hold.

(d) $A=\left(a_{n k}\right) \in(c, c)$ if and only if (1), (3) and (5) hold.

(e) $A=\left(a_{n k}\right) \in\left(c_{0}, l_{\infty}\right)$ if and only if (1) holds.

(f) $A=\left(a_{n k}\right) \in\left(c_{0}, l_{1}\right)$ if and only if (6) holds.

Lets pay attention that when $\alpha=1$ in (1), (2) and (5), we get the well known Silverman-Toeplitz Theorem in [15] which gives the necessary and sufficient conditions for a matrix to be regular.

Let $\left\{p_{n}\right\}$ be a sequence of non-negative numbers which are not all 0 and put

$$
P_{n}:=p_{1}+p_{2}+\ldots+p_{n} ; \quad p_{1}>0 .
$$


Definition 1.2. 14 The transformation

$$
\tilde{t}_{n}:=\frac{p_{n} x_{1}+p_{n-1} x_{2}+\ldots+p_{1} x_{n}}{P_{n}}
$$

is called the Nörlund mean of $\left(x_{k}\right)$. Matrix representation of Nörlund mean $(N, p)$ is given as follow:

$$
\tilde{a}_{n k}:=\left\{\begin{array}{cc}
\frac{p_{n-k+1}}{P_{n}}, & k \leq n, \\
0, & \text { otherwise. }
\end{array}\right.
$$

Definition 1.3. [14] The transformation given by

$$
t_{n}:=\frac{p_{1} x_{1}+p_{2} x_{2}+\ldots+p_{n} x_{n}}{P_{n}}
$$

is called the Riesz mean of $\left(x_{k}\right)$. Matrix representation of the $(R, p)$ is given by

$$
a_{n k}:=\left\{\begin{array}{cc}
\frac{p_{k}}{P_{k}}, & k \leq n, \\
0, & \text { otherwise. }
\end{array}\right.
$$

The transformations $(N, p)$ and $(R, p)$ are regular if and only if $P_{n} \rightarrow \infty, \quad(n \rightarrow \infty)$ [14].

The Cesàro mean $(C, 1)$ is a special case of both the Nörlund and the Riesz means with $p_{n}=1$ for all $n$ and its matrix representation is

$$
C_{n k}:=\left\{\begin{array}{lc}
\frac{1}{n}, & k \leq n, \\
0, & \text { otherwise }
\end{array}\right.
$$

Definition 1.4. [1] Let $\lambda=\{\lambda(n)\}$ be a strictly increasing sequence of positive integers. $C_{\lambda}$-transformation of a sequence $x=\left(x_{n}\right)$ is defined by

$$
t_{n}:=\frac{x_{1}+x_{2}+\ldots+x_{\lambda(n)}}{\lambda(n)}
$$

Definition 1.5. [14] A matrix $A=\left(a_{m, k}\right)$ is called $(M)$ matrix if $A$ is triangular and the inequality

$$
\left|\sum_{k=1}^{n} a_{m, k} x_{k}\right| \leq K\left|\sum_{k=1}^{n} a_{n^{\prime}, k} x_{k}\right|
$$

holds for some $n^{\prime}, n^{\prime}=n^{\prime}(n) \quad\left(0 \leq n^{\prime} \leq n\right), \quad(n=1,2,3, \ldots)$ and for all $m(m \geq n)$.

The number $n^{\prime}$ depends on $n$ and $\left(x_{n}\right)$ but not $m$. The regular matrices are not contain to the class $(M)$.

Consider Cesàro matrix, if $k<n+1$, then

$$
\frac{1}{n+1} \sum_{m=0}^{k} s_{m} \leq \frac{1}{k+1} \sum_{m=0}^{k} s_{m}
$$

holds. So, Cesàro matrix is an $(M)$ matrix [14].

Theorem 1.6. 14 Let $A=\left(a_{m, n}\right)$ and $B=\left(b_{m, n}\right)$ be regular triangular matrices and $A$ be an $(M)$ matrix. Then, if

$$
\sum_{n=1}^{m}\left|\frac{b_{m, n}}{a_{m, n}}-\frac{b_{m, n+1}}{a_{m, n+1}}\right|<M
$$

it follows that $B$ is a stronger than $A$. 
Theorem 1.7. 14 If the matrix $A=\left(a_{m, n}\right)$ is triangular and satisfies the conditions:

$$
a_{m, k}=0, \quad 0 \leq \frac{a_{m, k}}{a_{n, k}} \leq K
$$

and

$$
\frac{a_{m, k}}{a_{n, k}} \geq \frac{a_{m, k+1}}{a_{n, k+1}}
$$

hold for $0 \leq k \leq n \leq m$, then $A$ is an $(M)$ matrix.

The sequence of Fibonacci numbers is

$$
\left(f_{n}\right)=(0,1,1,2,3,5,8,13,21,34,55,89,144,233, \ldots) .
$$

It is clear from the elements of $\left(f_{n}\right)$ that $f_{0}=0, f_{1}=1$ and recurrence formula

$$
f_{n}:=f_{n-1}+f_{n-2}, \quad n \geq 2,
$$

holds.

Some properties of Fibonacci numbers are given as follows:

$$
\begin{aligned}
& \lim _{n \rightarrow \infty} \frac{f_{n+1}}{f_{n}}=\frac{1+\sqrt{5}}{2} \text { (golden ratio), } \\
& F_{n}:=\sum_{k=1}^{n} f_{k}=f_{n+2}-1, \quad n \in \mathbb{N} \\
& \sum_{k=1}^{\infty} \frac{1}{f_{k}} \text { converges, } \\
& \sum_{k=1}^{n}{f_{k}}^{2}=f_{n} f_{n+1} .
\end{aligned}
$$

Fibonacci sequence have may applications in science, art and architecture. In recent years, due to the interesting structure of the Fibonacci sequence, it has been found some application in the theory of summability and sequence spaces ([2], 6], [3], 4], [13]).

For example; M. Karakaş in [12] by considering the Fibonacci matrix as follows:

$$
f_{n k}:=\left\{\begin{array}{cl}
\frac{f_{2 k}}{f_{2 n+1}-1}, & 1 \leq k \leq n, \\
0, & \text { otherwise }
\end{array}\right.
$$

established the sequence spaces $c_{0}(F), c(F), l_{\infty}(F)$ and $l_{p}(F)(1 \leq p<\infty)$. follows:

Also, this type of sequence spaces were defined by E. E. Kara in [9] with a different type Fibonacci matrix as

$$
f_{n k}:=\left\{\begin{array}{cc}
-\frac{f_{n+1}}{f_{n}}, & k=n-1, \\
\frac{f_{n}}{f_{n+1}}, & k=n, \\
0, & 0 \leq k<n-1 \text { or } k>n .
\end{array}\right.
$$

In [9], Fibonacci difference sequence spaces $l_{p}(F),(1 \leq p<\infty)$ and $l_{\infty}(F)$ are defined as:

$$
\begin{aligned}
& l_{p}(F):=\left\{x=\left(x_{k}\right) \in w: \sum_{n}\left|\frac{f_{n}}{f_{n+1}} x_{n}-\frac{f_{n+1}}{f_{n}} x_{n-1}\right|^{p}<\infty\right\}, \\
& l_{\infty}(F):=\left\{x=\left(x_{k}\right) \in w: \sup _{n \in \mathbb{N}}\left|\frac{f_{n}}{f_{n+1}} x_{n}-\frac{f_{n+1}}{f_{n}} x_{n-1}\right|<\infty\right\} .
\end{aligned}
$$


Later, in [5], the spaces $c_{0}(F)$ and $c(F)$ were defined by using Fibonacci difference matrix in 10 as:

$$
c_{0}(F):=\left\{x=\left(x_{k}\right) \in w: \lim _{n \rightarrow \infty}\left(\frac{f_{n}}{f_{n+1}} x_{n}-\frac{f_{n+1}}{f_{n}} x_{n-1}\right)=0\right\},
$$

and

$$
c(F):=\left\{x=\left(x_{k}\right) \in w: \exists l \in \mathbb{C} \text { such that } \lim _{n \rightarrow \infty}\left(\frac{f_{n}}{f_{n+1}} x_{n}-\frac{f_{n+1}}{f_{n}} x_{n-1}\right)=l\right\} .
$$

Furthermore, E. E. Kara and M. Ilkkan in [10] defined a matrix $T=\left(t_{n k}\right)$ as follows:

$$
t_{n k}:=\left\{\begin{array}{cc}
t_{n}, & 1 \leq k=n, \\
-\frac{1}{t_{n}}, & k=n-1, \\
0, & 0 \leq k<1 \text { or } k>n .
\end{array}\right.
$$

For $t_{n}=\frac{f_{n}}{f_{n+1}}$ in the matrix $T=\left(t_{n k}\right)$, the matrix given in 10 has been obtained. Thus, a generalization of Fibonacci difference sequence has been given. In [11, using the matrix $T$, the sequence spaces $c_{0}(T)$ and $c(T)$ were defined and $\alpha, \beta$ and $\gamma$ duals of these spaces were determined.

In the light of these studies, here we define a new Fibonacci matrix $F=\left(f_{n k}\right)$ which is different $(9)$ and $(10)$ as follows:

$$
f_{n k}:=\left\{\begin{array}{cc}
\frac{f_{k}}{f_{n+2}-1}, & 1 \leq k \leq n, \\
0, & k>n .
\end{array}\right.
$$

It is clear from (11) that the Fibonacci matrix $F=\left(f_{n k}\right)$ is a lower triangle matrix.

In this paper, some relations between the matrix in $F=\left(f_{n k}\right)$ and some other known matrices has been compared and some inclusion results are given. Sequence spaces $c_{0}(F), c(F), l_{p}(F) \quad(1 \leq p<\infty)$ and $l_{\infty}(F)$ are defined.

It is shown that the sequence space $X\left(=c_{0}, c, l_{p}(1 \leq p<\infty)\right.$ or $\left.l_{\infty}\right)$ is isometrically isomorphic with the sequence space $X(F)$, respectively.

Finally, $\alpha, \beta$ and $\gamma$ duals of the spaces $c_{0}(F), c(F), l_{p}(F) \quad(1 \leq p<\infty)$ and $l_{\infty}(F)$ have been determined.

\section{Inclusion results for the matrix $F:=\left(f_{n k}\right)$}

In this section, $F$-transformation of $x=\left(x_{k}\right)$ is going to defined and its relationship with other well known matrix transformations (such as Nörlund, Riesz and Cesàro) will be examined.

Definition 2.1. Let $F=\left(f_{n k}\right)$ be a Fibonacci matrix given in (11). A real valued sequence $y=\left(y_{n}\right)$ is called F-transform of a sequence $x=\left(x_{n}\right)$ if

$$
y_{n}:=F\left(x_{n}\right)=\frac{1}{f_{n+2}-1} \sum_{k=1}^{n} f_{k} x_{k}
$$

exists for all $n \in \mathbb{N}$.

Definition 2.2. Let $x=\left(x_{n}\right)$ be a real valued sequence and $l \in \mathbb{R}$. It is called $F$-convergent to $l$ if $\left(F\left(x_{n}-l\right)\right)_{n \in \mathbb{N}}$ convergent to 0 .

Theorem 2.3. The Fibonacci matrix $F=\left(f_{n k}\right)$ is a regular summability method if and only if $f_{n+2}-1 \rightarrow \infty$ when $n \rightarrow \infty$. 
Proof Assume that $F=\left(f_{n k}\right)$ is a regular summability method. Then, from Silverman-Toeplitz theorem in [15].

$$
\lim _{n \rightarrow \infty} f_{n k}=\lim _{n \rightarrow \infty} \frac{f_{k}}{f_{n+2}-1}=0
$$

must be satisfied. So, $f_{n+2}-1 \rightarrow \infty, n \rightarrow \infty$.

Conversely, suppose $f_{n+2}-1 \rightarrow \infty$ when $n \rightarrow \infty$. We should check the conditions of Silverman- Toeplitz Theorem. Then,

$$
\sum_{k=1}^{\infty} \frac{f_{k}}{f_{n+2}-1}=\sum_{k=1}^{n} \frac{f_{k}}{f_{n+2}-1}=1
$$

For every $k \in \mathbb{N}$, by hypothesis

$$
\lim _{n \rightarrow \infty} f_{n k}=\lim _{n \rightarrow \infty} \frac{f_{k}}{f_{n+2}-1}=0
$$

and

$$
\lim _{n \rightarrow \infty} \sum_{k=1}^{\infty} f_{n k}=\lim _{n \rightarrow \infty} \sum_{k=1}^{n} f_{n k}=\lim _{n \rightarrow \infty} \sum_{k=1}^{n} \frac{f_{k}}{f_{n+2}-1}=1
$$

hold. Thus, $F=\left(f_{n k}\right)$ is a regular summability method.

Theorem 2.4. The Fibonacci matrix $F=\left(f_{n k}\right)$ is an $(M)$ matrix.

Proof It is enough that the matrix $F$ satisfies $(7)$ and (8). The following inequalities

$$
0 \leq \frac{f_{k}}{f_{m+2}-1} \cdot \frac{f_{n+2}-1}{f_{k}}=\frac{f_{n+2}-1}{f_{m+2}-1} \leq \frac{f_{n+2}}{f_{m+2}} \leq 1
$$

and

$$
\frac{\frac{f_{k+1}}{f_{m+2}-1}}{\frac{f_{k+1}}{f_{n+2}-1}}=\frac{f_{n+2}-1}{f_{m+2}-1} \cdot \frac{f_{k}}{f_{k}}=\frac{f_{k}}{f_{m+2}-1} \cdot \frac{f_{n+2}-1}{f_{k}}=\frac{a_{m, k}}{a_{n, k}}
$$

holds. Thus, the Fibonacci matrix $F$ is $(M)$ matrix.

Definition 2.5. Let $a=\left(a_{n}\right)$ and $b=\left(b_{n}\right)$ be two real valued sequences. They are called equivalent if there exist positive real numbers $m$ and $M$ such that inequality

$$
m \cdot a_{n} \leq b_{n} \leq M \cdot a_{n}
$$

holds for all $n \in \mathbb{N}$. It is denoted by $a \asymp b$.

The following Theorem gives a relation between $F$ and $(R, p)$ :

Theorem 2.6. Let $F=\left(f_{n k}\right)$ be a Fibonacci matrix, $x=\left(x_{n}\right)$ be a real valued sequence. Then, $x_{n} \rightarrow l(F)$ if and only if $x_{n} \rightarrow l(R, p)$, for any sequence $\left(p_{n}\right)$ such that $p_{n} \asymp f_{n}$ for all $n \in \mathbb{N}$.

Proof Suppose that $x_{n} \rightarrow l(F)$. So, we have

$$
\lim _{n \rightarrow \infty} \frac{1}{f_{n+2}-1} \sum_{k=1}^{n} f_{k}\left(x_{k}-l\right)=0
$$

Under the assumption on $\left(p_{n}\right)$, following inequality

$$
\frac{1}{P_{n}} \sum_{k=1}^{n} p_{k}\left(x_{k}-l\right) \leq \frac{1}{P_{n}} \sum_{k=1}^{n} M . f_{k}\left(x_{k}-l\right) \leq \frac{M}{m} \frac{1}{f_{n+2}-1} \sum_{k=1}^{n} f_{k}\left(x_{k}-l\right)
$$


holds and by the same way we have also

$$
\frac{m}{M} \frac{1}{f_{n+2}-1} \sum_{k=1}^{n} f_{k}\left(x_{k}-l\right) \leq \frac{1}{P_{n}} \sum_{k=1}^{n} p_{k}\left(x_{k}-l\right) .
$$

Since $x_{k} \rightarrow l(F)$, then 13$)$ and $(13)$ give that

$$
\lim _{n \rightarrow \infty} \frac{1}{P_{n}} \sum_{k=1}^{n} p_{k}\left(x_{k}-l\right)=0 .
$$

This complete the proof.

Sufficiency of the Theorem 2.6 can be proven in the same analogy. So, it is omitted here.

Theorem 2.7. Let $A=\left(a_{n k}\right)$ be any regular matrix and assume that

$$
\sum_{k=1}^{n}\left|a_{n k}-f_{n k}\right| \rightarrow 0, \quad(n \rightarrow \infty)
$$

Then, $x_{n} \rightarrow l \quad(A)$ if and only if $x_{n} \rightarrow l(F)$, for any bounded sequence.

Proof Let $x=\left(x_{n}\right)$ be a bounded sequence. For any $n$, following inequality

$$
\begin{aligned}
\left|(A x)_{n}-(F x)_{n}\right| & =\left|\sum_{k=1}^{n} a_{n k} x_{k}-\sum_{k=1}^{n} f_{n k} x_{k}\right| \\
& \leq \sum_{k=1}^{n}\left|a_{n k}-f_{n k}\right|\left|x_{k}\right| \\
& \leq\|x\| \sum_{k=1}^{n}\left|a_{n k}-f_{n k}\right|
\end{aligned}
$$

holds. Therefore, if $x_{n} \rightarrow l(A)$, then we have

$$
\left|(F x)_{n}-l\right| \leq\left|(F x)_{n}-(A x)_{n}\right|+\left|(A x)_{n}-l\right| \rightarrow 0, \quad n \rightarrow \infty .
$$

Similarly, if $x_{n} \rightarrow l(F)$, then we have

$$
\left|(A x)_{n}-l\right| \leq\left|(A x)_{n}-(F x)_{n}\right|+\left|(F x)_{n}-l\right| \rightarrow 0, \quad n \rightarrow \infty .
$$

So, (14) and $(15)$ complete the proof.

Now let's define associate matrix $\tilde{F}=\left(\tilde{f}_{n k}\right)$ as follows:

$$
\tilde{f}_{n k}:=\left\{\begin{array}{cl}
\frac{f_{n-k}}{f_{n+2}-1}, & k \leq n \\
0, & k>n .
\end{array}\right.
$$

The matrix $\tilde{F}=\left(\tilde{f}_{n k}\right)$ can be expressed as a Nörlund type Fibonacci matrix while the matrix $F=\left(f_{n k}\right)$ can be expressed as a Riesz type Fibonacci matrix.

Next theorems is about $\tilde{F}$. But, firstly, we remind a Lemma in [14].

Lemma 2.8. If $\left(N, p_{n}\right)$ is a regular Nörlund matrix, the series $\sum_{n=1}^{\infty} p_{n} x^{n-1}$ and $\sum_{n=1}^{\infty} P_{n} x^{n-1}$ are convergent for all $x,|x|<1$. 
Since the matrix $\tilde{F}=\left(\tilde{f}_{n k}\right)$ is a Nörlund typed matrix, then the above lemma is valid for $\tilde{F}=\left(\tilde{f}_{n k}\right)$.

From the definition of the matrix $\tilde{F}=\left(\tilde{f}_{n k}\right)$, we can take $F_{n}$ instead of $\tilde{F}_{n}$.

Since the following series are convergent for all $|x|<1$,

$$
f(x):=\sum_{n=1}^{\infty} f_{n} x^{n-1}, \quad F(x):=\sum_{n=1}^{\infty} F_{n} x^{n-1},
$$

then, the following series

$$
\begin{aligned}
& k(x)=\frac{p(x)}{f(x)}=\frac{P(x)}{F(x)}, \quad k(x):=\sum_{n=1}^{\infty} k_{n} x^{n-1}, \\
& h(x)=\frac{f(x)}{p(x)}=\frac{F(x)}{P(x)}, \quad h(x):=\sum_{n=1}^{\infty} h_{n} x^{n-1} .
\end{aligned}
$$

are also convergent for all $x,|x|<1$.

Theorem 2.9. $\left(N, p_{n}\right) \subseteq(\tilde{F})$ if and only if there exists $M>0$ such that

$$
\left|k_{1}\right| P_{n}+\left|k_{2}\right| P_{n-1}+\ldots+\left|k_{n}\right| P_{1} \leq M . F_{n},
$$

for every $n$ holds and

$$
\lim _{n \rightarrow \infty} \frac{k_{n}}{F_{n}}=0
$$

satisfied.

Proof The idea in [14 will be used in the proof. For sufficiency: Let $\left(u_{n}\right)$ and $\left(v_{n}\right)$ be the $(N, p)$ and $(\tilde{F})$ transformattion of a real valued sequence $\left(s_{n}\right)$, respectively. Then, we have following equality

$$
\begin{aligned}
\sum_{n=1}^{\infty} F_{n} v_{n} x^{n-1}= & \sum_{n=1}^{\infty} F_{n} \frac{\left(f_{n} s_{1}+f_{n-1} s_{2}+\ldots+f_{1} s_{n}\right)}{F_{n}} x^{n-1} \\
= & \left(f_{1} s_{1}\right) x^{0}+\left(f_{2} s_{1}+f_{1} s_{2}\right) x^{1}+\left(f_{3} s_{1}+f_{2} s_{2}+f_{1} s_{3}\right) x^{2}+\ldots \\
+ & \left(f_{n} s_{1}+f_{n-1} s_{2}+\ldots+f_{1} s_{n}\right) x^{n-1}+\ldots \\
= & s_{1}\left(f_{1} x^{0}+f_{2} x^{1}+f_{3} x^{2}+\ldots\right)+s_{2}\left(f_{1} x+f_{2} x^{2}+f_{3} x^{3}+\ldots\right) \\
+ & s_{3}\left(f_{1} x^{2}+f_{2} x^{3}+f_{3} x^{4}+\ldots\right)+\ldots+s_{n}\left(f_{1} x^{n-1}\right)+\ldots \\
= & s_{1} x^{0}\left(f_{1} x^{0}+f_{2} x^{1}+f_{3} x^{2}+\ldots+f_{n} x^{n-1}\right)+s_{2} x^{1}\left(f_{1} x^{0}+f_{2} x^{1}+\right. \\
& \left.f_{3} x^{2}+\ldots+f_{n-1} x^{n-2}\right)+\ldots+s_{n} x^{n-1}\left(f_{1} x^{0}\right)+\ldots \\
= & \left(\sum_{n=1}^{\infty} s_{n} x^{n-1}\right)\left(\sum_{n=1}^{\infty} f_{n} x^{n-1}\right)=s(x) f(x) .
\end{aligned}
$$

Using the same method, we get the equality

$$
\sum_{n=1}^{\infty} P_{n} u_{n} x^{n-1}=s(x) p(x) .
$$

By the hypothesis, we know that

$$
f(x)=k(x) p(x)
$$

and

$$
f(x) s(x)=k(x) p(x) s(x) .
$$


satisfied.

By the Cauchy product of series and the equalities (17) and (18), we have

$$
\sum_{n=1}^{\infty} F_{n} v_{n} x^{n-1}=\sum_{n=1}^{\infty} \sum_{m=1}^{n} k_{n-m+1} P_{m} u_{m} x^{n-1}
$$

and the equality of these series gives that

$$
F_{n} v_{n}=k_{n} P_{1} u_{1}+k_{n-1} P_{2} u_{2}+\ldots+k_{1} P_{n} u_{n}
$$

for all $n \in \mathbb{N}$. So,

$$
\begin{gathered}
v_{n}=\sum_{n=1}^{\infty} a_{n m} u_{m}, \\
a_{n m}=\left\{\begin{array}{cc}
\frac{k_{n-m+1} P_{m}}{F_{n}}, & m \leq n, \\
0, & m>n .
\end{array}\right.
\end{gathered}
$$

The matrix $\left(a_{n m}\right)$ is a regular matrix. Indeed,

$$
\begin{gathered}
\lim _{n \rightarrow \infty} a_{n m}=\lim _{n \rightarrow \infty} \frac{k_{n-m+1} P_{m}}{F_{n}}=\lim _{n \rightarrow \infty} \frac{k_{n-m+1} P_{m}}{F_{n-m+1}}=0, \\
\sum_{m=1}^{\infty}\left|a_{n m}\right|=\frac{\left|k_{1}\right| P_{n}+\ldots+\left|k_{n}\right| P_{1}}{F_{n}} \leq M \quad(n \in \mathbb{N})
\end{gathered}
$$

and

$$
\lim _{n \rightarrow \infty} \sum_{m=1}^{n} a_{n m}=\frac{k_{1} P_{n}+\ldots+k_{n} P_{1}}{F_{n}}=\frac{F_{n}}{F_{n}}=1 .
$$

Thus, from the regularity of $\left(a_{n m}\right)$ the sufficiency of the Theorem 2.9 is proved. The necessary part of Theorem 2.9 can also be shown easily with the conditions given in the theorem.

Now, let us consider a sequence $\left(g_{n}\right)$ defined by $g_{n}:=g_{n-1}+g_{n-2}, \quad n \geq 2$ such that $g_{0}=a$ and $g_{1}=b$ for any positive $a, b \in \mathbb{R}$. This sequence can be called Fibonacci type sequence. So, Fibonacci typed matrix $G=\left(g_{n k}\right)$ is defined as

$$
g_{n k}:=\left\{\begin{array}{cl}
\frac{g_{n-k}}{G_{n}}, & k \leq n, \\
0 & k>n
\end{array}\right.
$$

where $G_{n}:=g_{0}+g_{1}+\ldots+g_{n}=g_{n+2}-b$ and the matrix $G=\left(g_{n k}\right)$ is a regular matrix.

Theorem 2.10. For any two regular $\left(F, f_{n}\right)$ and $\left(G, g_{n}\right)$, there exists a regular $\left(H, h_{n}\right)$ such that $\left(H, h_{n}\right) \supseteq$ $\left(F, f_{n}\right)$ and $\left(H, h_{n}\right) \supseteq\left(G, g_{n}\right)$.

Proof Denote by $\left\{t_{n}^{1}\right\}$ and $\left\{t_{n}^{2}\right\}$ the $\left(F, f_{n}\right)$ and $\left(G, g_{n}\right)$ transformation of the sequence $\left\{s_{n}\right\}$, respectively. Let a sequence $\left\{h_{n}\right\}$ as follows:

$$
h_{n}:=f_{n} g_{0}+f_{n-1} g_{1}+\ldots+f_{0} g_{n},
$$


for all $n \in \mathbb{N}$. The sequence $\left\{s_{n}\right\}$ is transformed into $\left\{t_{n}\right\}$ by $\left(H, h_{n}\right)$, where:

$$
\begin{aligned}
t_{n} & =\frac{h_{1} s_{n}+h_{2} s_{n-1}+\ldots+h_{n} s_{1}}{h_{1}+h_{2}+\ldots+h_{n}} \\
& =\frac{\left(f_{1} g_{0}+f_{0} g_{1}\right) s_{n}+\left(f_{2} g_{0}+f_{1} g_{1}+f_{0} g_{2}\right) s_{n-1}+\ldots+\left(f_{n} g_{0}+\ldots+f_{0} g_{n}\right) s_{1}}{\left(f_{1} g_{0}+f_{0} g_{1}\right)+\left(f_{2} g_{0}+f_{1} g_{1}+f_{0} g_{2}\right)+\ldots+\left(f_{n} g_{0}+\ldots+f_{0} g_{n}\right)} \\
& =\frac{f_{1}\left(g_{0} s_{n}+\ldots+g_{n-1} s_{1}\right)+f_{2}\left(g_{0} s_{n-1}+\ldots+g_{n-2} s_{1}\right) \ldots+f_{n}\left(g_{0} s_{1}\right)}{f_{1}\left(g_{0}+\ldots+g_{n}\right)+\ldots+f_{n} g_{0}} \\
& =\frac{f_{1} G_{n}\left(\frac{g_{0} s_{n}+\ldots+g_{n-1} s_{1}}{G_{n}}\right)+\ldots+f_{n} G_{0}\left(\frac{g_{0} s_{1}}{G_{0}}\right)}{f_{1} G_{n}+\ldots+f_{n} G_{0}} \\
& =\frac{f_{1} G_{n} t_{n}^{2}+\ldots+f_{n} G_{0} t_{0}^{2}}{f_{1} G_{n}+\ldots+f_{n} G_{0}}
\end{aligned}
$$

Clearly, $\left(H, h_{n}\right)$ mean of $\left(s_{n}\right)$ is the $A=\left(a_{m n}\right)$ transformation of $\left\{t_{n}^{2}\right\}$, where

$$
a_{n m}:=\left\{\begin{array}{cl}
\frac{f_{n-m} G_{m}}{\sum_{k=0}^{n} f_{n-k} G_{k}}, & m \leq n, \\
0, & m>n .
\end{array}\right.
$$

Since the equality

$$
\sum_{m=0}^{n} a_{n m}=\sum_{m=0}^{n}\left|a_{n m}\right|=1
$$

satisfies and for $\sum_{k=1}^{n} f_{n-k} G_{k}>K F_{n}, \quad\left(K \geq g_{0}>0\right)$ and for all fixed $n$, the inequality

$$
0 \leq \lim _{n \rightarrow \infty} \frac{f_{n-m} G_{m}}{\sum_{k=0}^{n} f_{n-k} G_{k}}<\lim _{n \rightarrow \infty} \frac{f_{n-k} G_{k}}{K F_{n}}=0
$$

satisfies, then $A=\left(a_{n m}\right)$ is a regular matrix. this implies, if $\left\{t_{n}^{2}\right\}$ converges, then $\left\{t_{n}\right\}$ converges to the same value. This implies that $\left(H, h_{n}\right) \supseteq\left(G, g_{n}\right)$ and by similar way, $\left(H, h_{n}\right) \supseteq\left(F, f_{n}\right)$ can be shown.

Definition 2.11. 14] The matrices $A$ and $B$ are said to be equivalent if the inclusions $A \supseteq B$ and $B \supseteq A$ hold.

Theorem 2.12. The regular means $\left(F, f_{n}\right)$ and $\left(G, g_{n}\right)$ are equivalent if and only if the associated series $\sum_{n=1}^{\infty}\left|k_{n}\right|$ and $\sum_{n=1}^{\infty}\left|h_{n}\right|$ are converge.

Proof Suppose that $\left(F, f_{n}\right)$ and $\left(G, g_{n}\right)$ are equivalent. Then, there is an $M>0$ such that $0<k_{0} F_{n}<M G_{n}$ and $0<h_{0} G_{n}<M F_{n}$ hold for all $n \in \mathbb{N}$ and the sequence $\left(\frac{F_{n}}{G_{n}}\right)$ and $\left(\frac{G_{n}}{F_{n}}\right)$ are both bounded. In continuation of the proof, if it is followed way as in [14, then the proof is completed.

Definition 2.13. Let $\lambda=\lambda(n)$ be a strictly increasing sequence of positive integers. $F_{\lambda}$-transform of a sequence $x=\left(x_{n}\right)$ is defined by

$$
t_{n}:=\frac{f_{1} x_{1}+f_{2} x_{2}+\ldots+f_{\lambda(n)} x_{\lambda(n)}}{f_{\lambda(n)+2}-1} .
$$

Let's remind notation $o(1)$ before giving next theorem:

$f(n)=o(g(n))$ means for all $c>0$ there exists some $k>0$ such that $0 \leq f(n)<c g(n)$ for all $n \geq k$. The value of $k$ must not depend on $n$, but may depend on $c$.

Theorem 2.14. Let $\lambda=\{\lambda(n)\}$ and $\mu=\{\mu(n)\}$ be a strictly increasing sequences of $\mathbb{N}$. If $\lim _{n \rightarrow \infty} \frac{f_{\lambda(n)+2}-1}{f_{\mu(n)+2}-1}=$ 1 , then $F_{\lambda}$ is equivalent to $F_{\mu}$ on $l_{\infty}$. 
Proof Let $x=\left(x_{n}\right)$ be a bounded sequence and $M(n)=\max \{\{\lambda(n)\},\{\mu(n)\}\}, \operatorname{m}(n)=\min \{\{\lambda(n)\},\{\mu(n)\}\}$. Since $\lim _{n \rightarrow \infty} \frac{f_{\lambda(n)+2}-1}{f_{\mu(n)+2}-1}=1$ and $\lim _{n \rightarrow \infty} \frac{f_{m(n)+2}-1}{f_{M(n)+2}-1}=1$, then, for any $n$,

$$
\begin{aligned}
& \left|\left(F_{\lambda} x\right)_{n}-\left(F_{\mu} x\right)_{n}\right|=\left|\frac{1}{f_{\mu(n)+2}-1} \sum_{k=1}^{\mu(n)} f_{k} x_{k}-\frac{1}{f_{\lambda(n)+2}-1} \sum_{k=1}^{\lambda(n)} f_{k} x_{k}\right| \\
= & \left|\frac{1}{f_{M(n)+2}-1} \sum_{k=1}^{M(n)} f_{k} x_{k}-\frac{1}{f_{m(n)+2}-1} \sum_{k=1}^{m(n)} f_{k} x_{k}\right| \\
= & \left|\frac{1}{f_{M(n)+2}-1} \sum_{k=1}^{m(n)} f_{k} x_{k}+\frac{1}{f_{M(n)+2}-1} \sum_{k=m(n)+1} f_{k} x_{k}-\frac{1}{f_{m(n)+2}-1} \sum_{k=1}^{m(n)} f_{k} x_{k}\right| \\
= & \left|\sum_{k=1}^{m(n)} f_{k} x_{k}\left(\frac{1}{f_{M(n)+2}-1}-\frac{1}{f_{m(n)+2}-1}\right)+\frac{1}{f_{M(n)+2}-1} \sum_{k=m(n)+1} f_{k} x_{k}\right| \\
\leq & \|x\|_{\infty}\left(\sum_{k=1}^{m(n)} f_{k}\left|\frac{f_{m(n)+2}-f_{M(n)+2}}{\left(f_{M(n)+2}-1\right)\left(f_{m(n)+2}-1\right)}\right|+\sum_{k=m(n)+1} f_{k}\left|\frac{1}{f_{M(n)+2}-1}\right|\right) \\
\leq & \|x\|_{\infty}\left(\frac{\left(f_{M(n)+2}-f_{m(n)+2}\right)\left(f_{m(n)+2}-1\right)}{\left(f_{M(n)+2}-1\right)\left(f_{m(n)+2}-1\right)}+\frac{\left(f_{M(n)+2}-f_{m(n)+2}\right)}{f_{M(n)+2}-1}\right) \\
\leq & 2\|x\|_{\infty}\left(\frac{\left(f_{M(n)+2}-1\right)-\left(f_{m(n)+2}-1\right)}{\left(f_{M(n)+2}-1\right)}\right) \\
\leq & 2\|x\|_{\infty}\left(1-\frac{f_{m(n)+2}-1}{f_{M(n)+2}-1}\right)=o(1)
\end{aligned}
$$

holds. Thus, if $x$ is $F_{\lambda}$-summable to $L$, then we have

$$
0 \leq\left|\left(F_{\mu} x\right)_{n}-L\right| \leq\left|\left(F_{\mu} x\right)_{n}-\left(F_{\lambda} x\right)_{n}\right|+\left|\left(F_{\lambda} x\right)_{n}-L\right|=o(1)+o(1)=o(1) .
$$

Similarly, if $x$ is $F_{\mu}$-summable to $L$, then we have

$$
0 \leq\left|\left(F_{\lambda} x\right)_{n}-L\right| \leq\left|\left(F_{\lambda} x\right)_{n}-\left(F_{\mu} x\right)_{n}\right|+\left|\left(F_{\mu} x\right)_{n}-L\right|=o(1)+o(1)=o(1) .
$$

This complete the proof of theorem.

Theorem 2.15. Cesàro matrix is stronger than Fibonacci matrix $F=\left(f_{n k}\right)$.

Proof We should check that the inequality in Theorem 1.6 holds. For this, if we take $C_{n k}$ (Cesàro matrix) instead of $B$ and $F$ instead of $A$, we have

$$
\begin{aligned}
\sum_{k=1}^{n}\left|\frac{f_{n+2}-1}{n f_{k}}-\frac{f_{n+2}-1}{n f_{k+1}}\right| & =\sum_{k=1}^{n} \frac{f_{n+2}-1}{n}\left(\frac{1}{f_{k}}-\frac{1}{f_{k+1}}\right) \\
& =\frac{f_{n+2}-1}{n} \sum_{k=1}^{n}\left(\frac{1}{f_{k}}-\frac{1}{f_{k+1}}\right) .
\end{aligned}
$$

It is known that $n \leq f_{n+2}-1$ holds for all $n \in \mathbb{N}$. So, the following inequality

$$
\begin{aligned}
\frac{f_{n+2}-1}{n} \sum_{k=1}^{n}\left(\frac{1}{f_{k}}-\frac{1}{f_{k+1}}\right) & \leq \sum_{k=1}^{n}\left(\frac{1}{f_{k}}-\frac{1}{f_{k+1}}\right) \\
& \leq\left(\frac{1}{f_{1}}-\frac{1}{f_{2}}+\frac{1}{f_{2}}-\frac{1}{f_{3}}+\ldots+\frac{1}{f_{n}}-\frac{1}{f_{n+1}}\right) \leq 1-\frac{1}{f_{n+1}}
\end{aligned}
$$

satisfied. This inequality gives that the condition of Theorem 1.6 is satisfied. Thus, $F \subset C_{n k}$.

Remark 2.16. The converse of Theorem 2.15 is not true, in general. 
Let us consider $x=\left(x_{n}\right)$ as

$$
x_{n}:=\frac{(-1)^{n}}{n}
$$

The sequence $\left(C_{n} x\right)=\left(\frac{1}{n} \sum_{k=1}^{n} \frac{(-1)^{k}}{k}\right)$ is convergent, but if we consider $F$-transformation of $\left(x_{n}\right)$, then the sequence $\left(F_{n} x\right)=\frac{1}{f_{n+2}-1} \sum_{k=1}^{n} \frac{f_{k}\{-1\}^{k}}{k}$ is not convergent. Thus, it seen that $F \nsubseteq C_{n k}$.

\section{$3 \quad$ Fibonacci Sequence Spaces}

In this section, Fibonacci sequence spaces will be defined and some properties of them will be given.

$X$ denotes the sequence spaces $c_{0}, c, l_{\infty}$ or $l_{p}(1 \leq p<\infty)$ and

$$
\left(y_{n}\right):=(F x)_{n}=\left(\frac{1}{f_{n+2}-1} \sum_{k=1}^{n} f_{k} x_{k}\right)
$$

is F-transform of $x=\left(x_{n}\right)$.

Definition 1. The set

$$
X(F)=\left\{\left(x_{n}\right) \in w:\left(y_{n}\right) \in X\right\}
$$

is called Fibonacci sequence space.

Theorem 3.1. Let $X$ be a sequence space. Then, $X(F)$ is a BK space with the following norms (i) If $X=c_{0}, c$ or $l_{\infty}$,

$$
\|x\|_{X(F)}:=\sup \left\{\left|y_{n}\right|: n \in \mathbb{N}\right\}
$$

(ii) If $X=l_{p},(1 \leq p<\infty)$

$$
\|x\|_{X(F)}=\left(\sum_{k=1}^{\infty}\left|y_{k}\right|^{p}\right)^{\frac{1}{p}}
$$

Proof Since the matrix $F$ is triangle, then the result of A. Wilansky in [16] gives that the space $X(F)$ is a BK space.

Theorem 3.2. The space $X(F)$ is isometrically isomorphic to the space $X$.

Proof We should find an isometric isomorphism between the spaces $X(F)$ and $X$. For this, let us define a function $P$ by

$$
P: X(F) \mapsto X, \quad P x=y
$$

where $y=\left(y_{n}\right)=(F x)_{n}=\frac{1}{f_{n+2}-1} \sum_{k=1}^{n} f_{k} x_{k}$.

It is clear that $P$ is a linear function. Since $P x=0$ implies $x=0$, then $P$ is an injective function.

Now, we must show that $P$ is surjective. If we consider a sequence $x=\left(x_{n}\right)$ as

$$
x_{n}=\frac{f_{k+2}-1}{f_{k}} y_{k}-\frac{f_{k+1}-1}{f_{k}} y_{k-1},
$$

then for every $y=\left(y_{n}\right) \in X$, there exists a sequence $x=\left(x_{n}\right) \in X(F)$ such that $P x=y$. So, the equality

$$
(F x)_{n}=\frac{1}{f_{n+2}-1} \sum_{k=1}^{n} f_{k} x_{k}=\frac{1}{f_{n+2}-1} \sum_{k=1}^{n}\left[\left(f_{k+2}-1\right) y_{k}-\left(f_{k+1}-1\right) y_{k-1}\right]=y_{n} .
$$

holds and it gives that $x \in X(F)$ as $F x \in X$. Hereby, $P$ is surjective.

Also, $P$ is norm preserving function, that is,

$$
\|P x\|_{X}=\|y\|_{X}=\|F x\|_{X}=\|x\|_{X(F)},
$$

for any $x \in X(F)$. So, $P$ is an isometry. 
Theorem 3.3. Let $X$ be any of the spaces $c_{0}$, $c$ and $l_{\infty}$. Then, the inclusion $X \subset X(F)$ holds.

Proof Since the matrix $F$ is regular, then the inclusion is clear for $X=c_{0}$ and $c$. Let us take $x=\left(x_{n}\right) \in l_{\infty}$, there is a constant $M>0$ such that $\left|x_{n}\right| \leq M$ for $n \in \mathbb{N}$. Then, following inequality holds:

$$
\left|F_{n}(x)\right|=\left|\frac{1}{f_{n+2}-1} \sum_{k=1}^{n} f_{k} x_{k}\right| \leq \frac{1}{f_{n+2}-1} \sum_{k=1}^{n} f_{k}\left|x_{k}\right| \leq \frac{M}{f_{n+2}-1} \sum_{k=1}^{n} f_{k} \leq M .
$$

This inequality gives that $x=\left(x_{n}\right) \in l_{\infty}(F)$.

Now, if we take $x=\left(x_{n}\right) \in l_{p} \quad(1 \leq p<\infty)$, then there exists a constant $M>0$ such that

$$
\|x\|_{p}^{p}=\sum_{n=1}^{\infty}\left|x_{n}\right|^{p}<M
$$

We should show that $x=\left(x_{n}\right) \in l_{p}(F)$. From the Hölder inequality and the definition of $\left(f_{n k}\right)$, we have

$$
\begin{aligned}
{\left[\sum_{k=1}^{n} \frac{f_{k}}{f_{n+2}-1}\left|x_{k}\right|\right]^{p} } & \leq\left[\left(\sum_{k=1}^{n}\left(\frac{f_{k}}{f_{n+2}-1}\right)^{q}\right)^{\frac{1}{q}} \cdot\left(\sum_{k=1}^{n}\left|x_{k}\right|^{p}\right)^{\frac{1}{p}}\right]^{p} \\
& \leq\left(\sum_{k=1}^{n}\left(\frac{f_{k}}{f_{n+2}-1}\right)^{q}\right)^{\frac{p}{q}} \cdot\left(\sum_{k=1}^{n}\left|x_{k}\right|^{p}\right)
\end{aligned}
$$

where $\frac{1}{p}+\frac{1}{q}=1$.

On the right side of the inequality, since

$$
\left(\sum_{k=1}^{n}\left(\frac{f_{k}}{f_{n+2}-1}\right)^{q}\right)^{\frac{p}{q}}=\left(\frac{f_{1}^{q}+f_{2}^{q}+\ldots+f_{n}^{q}}{\left(f_{1}+f_{2}+\ldots+f_{n}\right)^{q}}\right)^{\frac{p}{q}} \leq 1
$$

then we have $\left|F_{n}(x)\right|^{p} \leq \sum_{k=1}^{n}\left|x_{k}\right|^{p}$. It gives that

$$
\sum_{n=1}^{\infty}\left|F_{n}(x)\right|^{p} \leq \sum_{n=1}^{\infty}|x|^{p}<\infty
$$

Thus, it was shown that $x \in l_{p}(F)$.

Theorem 3.4. Let $F$ be Fibonacci matrix. Then, following inclusion

$$
c_{0}(F) \subset c(F) \subset l_{\infty}(F)
$$

strictly holds.

Proof From Theorem 3.3 inclusion is obvious. Let us consider $x=\left(x_{n}\right)=(1,1,1, \ldots)$. Since the matrix $\mathrm{F}$ is regular, $F_{n} x$ is convergent. So, $\left(x_{n}\right) \in c(F)$. On the other hand, $\left(F_{n} x\right) \notin c_{0}$ and $\left(x_{n}\right) \notin c_{0}(F)$.

If we take the sequence $\left(x_{n}\right)=\left(\frac{(-1)^{n}\left(f_{n+2}+f_{n+1}-1\right)}{f_{n}}\right)$, for all $n \in \mathbb{N}$. Then, we have

$$
F_{n}(x)=\frac{1}{f_{n+2}-1} \sum_{k=1}^{n} f_{k}(-1)^{k} \frac{\left(f_{k+2}+f_{k+1}-1\right)}{f_{k}} .
$$

We see that the sequence

$$
F_{n} x= \begin{cases}1, & n \text { is even } \\ 0, & n \text { is odd }\end{cases}
$$

is in $l_{\infty}$. So, $\left(x_{n}\right) \in l_{\infty}$. But, since $\left(F_{n} x\right) \notin c$, then $\left(x_{n}\right) \notin c(F)$. and when $n$ is odd, the sequence $\left(F_{n}(x)\right)$ converges to -1 . We see that $\left(x_{n}\right) \notin c(F)$, but the sequence $\left(x_{n}\right) \in l_{\infty}(F)$. 


\section{$4 \alpha, \beta$ and $\gamma$ Duals of the Sequence Spaces $X$}

In this section, we determine the $\alpha, \beta$ and $\gamma$ duals of the sequence spaces $c_{0}(F), c(F), l_{p}(F) \quad(1 \leq p<\infty)$ for the Fibonacci matrix $F$.

The duals $\alpha, \beta$ and $\gamma$ of $X$ can be given as follows [16]:

$$
\begin{aligned}
X^{\alpha} & =\bigcap_{\left(x_{n}\right) \in X}\left[x_{n}\right]^{\alpha}, \\
X^{\beta} & =\bigcap_{\left(x_{n}\right) \in X}\left[x_{n}\right]^{\beta}, \\
X^{\gamma} & =\bigcap_{\left(x_{n}\right) \in X}\left[x_{n}\right]^{\gamma},
\end{aligned}
$$

where $\left[x_{n}\right]^{\alpha}, \quad\left[x_{n}\right]^{\beta}$ and $\left[x_{n}\right]^{\gamma}$ are sets of the sequences $a=\left(a_{n}\right) \in w$ which satisfy

$$
\begin{aligned}
& \left|\sum a_{n} x_{n}\right| \text { is convergent, } \\
& \sum a_{n} x_{n} \text { is convergent, } \\
& \sum a_{n} x_{n} \text { is bounded }
\end{aligned}
$$

respectively. Let us consider the sets

$$
\begin{aligned}
& d_{1}=\left\{a=\left(a_{n}\right) \in w: \sup _{K \in F} \sum_{n}\left|\sum_{k \in K} \frac{f_{k}}{f_{n+2}-1} a_{k}\right|<\infty\right\} \\
& d_{2}=\left\{a=\left(a_{n}\right) \in w: \sup _{n \in \mathbb{N}} \sum_{k=1}^{n}\left|\sum_{j=k}^{n} \frac{f_{j}}{f_{n+2}-1} a_{j}\right|<\infty\right\} \\
& d_{3}=\left\{a=\left(a_{n}\right) \in w: \lim _{n \rightarrow \infty} \sum_{j=k}^{n} \frac{f_{j}}{f_{n+2}-1} a_{j} \text { exists for each } n \in \mathbb{N}\right\} \\
& d_{4}=\left\{a=\left(a_{n}\right) \in w: \lim _{n \rightarrow \infty} \sum_{k=1}^{n} \sum_{j=k}^{n} \frac{f_{j}}{f_{n+2}-1} a_{j} \text { exists }\right\} .
\end{aligned}
$$

Theorem 4.1. The following statements hold

(a) $\left\{c_{o}(F)\right\}^{\alpha}=\{c(F)\}^{\alpha}=d_{1}$.

(b) $\left\{c_{o}(F)\right\}^{\beta}=d_{2} \cap d_{3}$ and $\{c(F)\}^{\beta}=d_{2} \cap d_{3} \cap d_{4}$.

(c) $\left\{c_{o}(F)\right\}^{\gamma}=\{c(F)\}^{\gamma}=d_{2}$.

Proof As in [5] and [9], we define the matrix $T=\left(t_{n k}\right)$ as follows:

$$
t_{n k}:=\left\{\begin{array}{cc}
\frac{f_{k}}{f_{n+2}-1} a_{k}, & k \leq n \\
0, & k>n
\end{array} .\right.
$$

for a given sequence $a=\left(a_{n}\right) \in w$. On the other hand, we know that since $\left|\sum a_{n} x_{n}\right|<\infty$ for every $x=\left(x_{n}\right) \in$ $c_{0}(F), \quad c(F)$, then $\left(y_{n}\right)=(F x)_{n} \in c_{0}, \quad c(F)$.

By using the definition of $\alpha$ dual, we will show that $(T y)_{n} \in l_{1}$ for $\left(y_{n}\right) \in c_{0}, \quad c$.

Then, we have

$$
a_{n} x_{n}=\sum_{k=1}^{n} \frac{f_{k}}{f_{n+2}-1} y_{k} a_{k}=(T y)_{n} \quad(\forall n \in \mathbb{N}) .
$$

Thus, we see that $(T y)_{n} \in l_{1}$ for $\left(y_{n}\right) \in c_{0}, \quad c$ if and only if $\left(a_{n} x_{n}\right) \in l_{1}$ for $\left(x_{n}\right) \in c_{0}(F), \quad c(F)$. This implies that $a=\left(a_{n}\right) \in l_{1}$.

The other statements can be proved in a similar way.

For $1<p \leq \infty$, let us find $\alpha, \beta$ and $\gamma$ duals of the $l_{p}(F)$. 
Theorem 4.2. The $\alpha$ dual of the space $l_{p}(F)$ is the set

$$
d_{1}=\left\{x=\left(x_{n}\right) \in w: \sup _{K \in F} \sum_{n}\left|\sum_{k \in K} \frac{f_{k}}{f_{n+2}-1} x_{k}\right|^{q}<\infty\right\}
$$

and $\beta, \quad \gamma$ duals are $\left\{l_{p}(F)\right\}^{\beta}=d_{2} \cap d_{3}$ and $\left\{l_{\infty}(F)\right\}^{\beta}=d_{3} \cap d_{4}$ and $\left\{l_{p}(F)\right\}^{\gamma}=d_{3}$, respectively.

Theorem 4.3. Let $\left(x_{n}\right)$ and $\left(y_{n}\right)$ be two sequence and $\left(a_{n}\right) \in w$ be a dual sequence. If the sequences $\left(x_{n}\right)$ and $\left(y_{n}\right)$ are asymptotically equivalent,i.e, $\lim _{n \rightarrow \infty} \frac{x_{n}}{y_{n}}=1$, then $\left[x_{n}\right]^{\beta}=\left[y_{n}\right]^{\beta}$

Proof Suppose that $\lim _{n \rightarrow \infty} \frac{x_{n}}{y_{n}}=1$ and $\left(a_{n}\right)$ be arbitrary sequence in $\left[x_{n}\right]^{\beta}$. Then, for every $\left(x_{n}\right) \in X$, the $\operatorname{sum} \sum_{n_{1}}^{\infty} a_{n} x_{n}$ is convergent so that the partial sum sequence $\left(s_{n}\right)$ of converges to $s \in \mathbb{R}$. For every $\varepsilon>0$, there is an $n_{0} \in \mathbb{N}$ such that $\forall n \geq n_{0}$, we have

$$
\left|\sum_{k=1}^{n} a_{k} y_{k}-s\right|=\left|\sum_{k=1}^{n} a_{k} x_{k} \frac{y_{k}}{x_{k}}-s\right|<\varepsilon .
$$

Thus, it is shown that $\left(a_{n}\right) \in\left[y_{n}\right]^{\beta}$. It can be easily proved the second part of the theorem.

Remark 4.4. The converse of Theorem 4.3 is not true, in general.

Let $x=\left(x_{n}\right)=\left(\frac{1}{n}\right), y=\left(y_{n}\right)=\left(\frac{1}{n^{2}}\right)$ and $a_{n}=(-1)^{n}$, for $\forall n \in \mathbb{N}$. Since the series $\sum_{n=1}^{\infty}(-1)^{n} \frac{1}{n}$ and $\sum_{n=1}^{\infty}(-1)^{n} \frac{1}{n^{2}}$ are convergent, then $\left[x_{n}\right]^{\beta}=\left[y_{n}\right]^{\beta}$. On the other hand, it seen that

$$
\lim _{n \rightarrow \infty} \frac{\frac{1}{n}}{\frac{1}{n^{2}}} \neq 1
$$

\section{Conclusion}

Since $F=\left(f_{n k}\right)$ is a regular matrix, then the sequence $\left(F_{n} x\right)$ is convergent for a convergent sequence $\left(x_{n}\right)$. Thus, a sequence space containing the space $c$ is obtained and many studies with regular matrices can be reexamined for the $F$ matrix. Also, statistical convergence of the sequence $\left(F_{n} x\right)$ can be studied .

Lastly, if a arbitrary normed spaces $X$ is taken instead of the space $X=\left(c_{0}, c, l_{p}(1 \leq p<\infty)\right.$, $\left.l_{\infty}\right)$, then it is obtained generalization of the Fibonacci sequence space $X(F)$. Given results in this paper can be investigated for the general Fibonacci sequence space.

Also, in the paper [14], some properties of Fibonacci Frames, orthonormal bases and Fibonacci Riesz bases were studied and dual Fibonacci Frames were characterized. Results given in [7] can be re-examined for the Fibonacci matrix $F=\left(f_{n k}\right)$ defined in this paper.

ACKNOWLEDGEMENTS. The authors would like to thank the referees for their valuable comments and suggestions that extend to the paper.

\section{References}

[1] D.H. Armitage and I.J. Maddox, A New Type of Ceàro Mean, Analysis, 9 (1989), 195-204.

[2] F. Başar, Summability Theory and Its Applications, Bentham e-books, 410s. (2011).

[3] F. Başar and K. Kayaduman, On The Domain of The Fibonacci Difference Matrix, Mathematics, 7(2)(2019): 204. https://doi.org/10.3390/math7020204

[4] M. Başarır, F. Başar and E. E. Kara, On The Spaces of Fibonacci Difference Absolutely p-Summable, Null and Convergent Sequence, SJM, 12(2)(2016). https://doi.org/10.5644/sjm.12.2.04

[5] M. Başarır, F. Başar and E.E. Kara, On The Spaces of Fibonacci Diffrence Null and Convergent Sequences, arXiv:1309.0150v1 [math.FA], (2013). 
[6] A. Das, B. Hazarika and F. Başar, Some Fibonacci Sequence Spaces of Non-Absolute Type Derived From $\ell_{p}$ with $(1 \leq p \leq \infty)$ and Hausdorff Measure of Non-Compactness of Composition Operators, June (2017).

[7] M.A. Denghan and G. Talebi, Lower Bound for Matrices on the Fibonacci Sequence Spaces and its Applications in Frame Theory, Results Math, 72 (2017), 1087-1107. https://doi.org/10.1007/s00025-016-0632-x

[8] E.E. Kara, and M. Başarır, An Application of Fibonacci Numbers into Infinite Toeplitz Matrices, CJMS, 1 (1) (2012), 43-47.

[9] E.E Kara, Some Topological and Geometrical properties of new Banach Sequence Spaces, Journal of Inequalities and Appl. (38) (2013). https://doi.org/10.1186/1029-242x-2013-38

[10] E. E. Kara and M. Ilkkan, On Some Banach Sequence Spaces Derived by a New Band Matrix, Math. Comput. Sci. 9 (2015), 141-159. https://doi.org/10.9734/bjmcs/2015/17499

[11] E. E. Kara and M. Ilkkan, Some Properties of Generalized Fibonacci Sequence Spaces, Linear and Multilinear Algebra, 64(11) (2016), 2208-2223. https://doi.org/10.1080/03081087.2016.1145626

[12] M. Karakaş, A New Regular Matrix Defined By Fibonacci Numbers and Applications, BEU Journal of Science, 4(2) (2015), 205-210. https://doi.org/10.17798/beufen.78452

[13] V.A. Khan, E. E. Kara, H. Altaf, N. Khan and A. Mobeen, Intuitionistic Fuzzy I-Convergent Fibonacci Difference Sequence Spaces, Journal of Inequality and Appl,(2019) (1). https://doi.org/10.1186/s13660-019-2152-1

[14] G.M. Petersen, Regular Matrix Transformations, McGraw-Hill Publishing Company Limited, (1966).

[15] M. Stieglitz, and H. Tietz, Matriztransformationen von Folgenräumen Eine Ergebniübersicht, Mathematische Zeitschrift, 154 (1977), 1-16. https://doi.org/10.1007/bf01215107

[16] A. Wilansky, Summability Trough Functional Analysis, Elseiver Science Publishers B. V., (1984). 